\title{
Acoustic Power Measurements of Oscillating Flames
}

\author{
M. VALK \\ The University of Twente, Enschede, The Netherlands
}

\begin{abstract}
The acoustic power of an oscillating flame is measured. A turbulent premixed propane/air flame is situated near a pressure antinode of a standing wave in a laboratory combustion chamber. This standing wave is generated by a piston. The fluctuating heat release of the flame will supply acoustic power to the standing wave as postulated by Rayleigh. This flame acoustic power is obtained by setting up a power balance of the whole combustion chamber.

Experiments proved that it is possible to measure quantitatively the acoustic power and the frequency change as a function of relevant parameters. This frequency change is a second phenomenon described by Rayleigh. The acoustic power of the flame as a function of the amplitude of the fluctuating component of the mixture flow velocity at different frequencies can be measured.

Measurement techniques are described and results for frequencies ranging from 3 to $120 \mathrm{~Hz}$ are given.
\end{abstract}

\section{INTRODUCTION}

The combustion driven oscillation is a well-known phenomenon, described in many papers and textbooks (see, e.g., Refs [1] and [3]). These oscillations can be very loud and annoying. The conditions under which they will occur spontaneously can be described in terms of the transfer functions of the flame, the combustion chamber, and the burner. It is known that these oscillations are brought on by a positive feedback mechanism, in which a normally steady supply of energy (the flame) is modulated through a passive feedback loop (the burner and combustion chamber).

This paper describes both the procedure and the experimental apparatus that make it possible to measure quantitatively the transfer function of a simple premixed turbulent propane-air flame. The input of the flame is the fluctuating component of the mixture flow rate. The output is the acoustic power produced by the flame. Experimental results are given for frequencies ranging from 3 to

Address: M. Valk, Technische Hogeschool Twente, Afdeling der Werktuigbouwkunde, Postbus 217, 7500 AE Enschede, The Netherlands.

Copyright $\odot 1981$ by The Combustion Institute

Published by Elsevier North Holland, Inc.

52 Vanderbilt Avenue, New York, NY 10017
$120 \mathrm{~Hz}$. The hypothesis that explains this acoustic power production in a combustion system was first proposed by Lord Rayleigh [2]. This hypothesis states that for a heat-driven oscillation to occur there must be a varying rate of heat release having a component in phase with the varying component of the pressure. The place of heat supply must also be other than a pressure node in the standing wave. The experimental results given in this paper confirm this hypothesis quantitatively.

\section{EXPERIMENTAL APPARATUS}

Figure 1 shows the experimental apparatus. The combustion chamber is a cylindrical tube with one open end. Inside this chamber a standing wave (quarter wave) is maintained at the natural frequency, driven by a piston at the closed end of the combustion chamber. Under this condition of resonance the piston velocity, $u_{\mathrm{c}}(t)$, is in phase with the sound pressure, $p_{\mathrm{c}}(t)$, of the standing wave. To control the amplitude of the sound pressure the piston displacement can be varied through the power supply to the electrodynamic exciter.

Two sound pressure transducers are placed in the combustion chamber. One transducer is situ- 


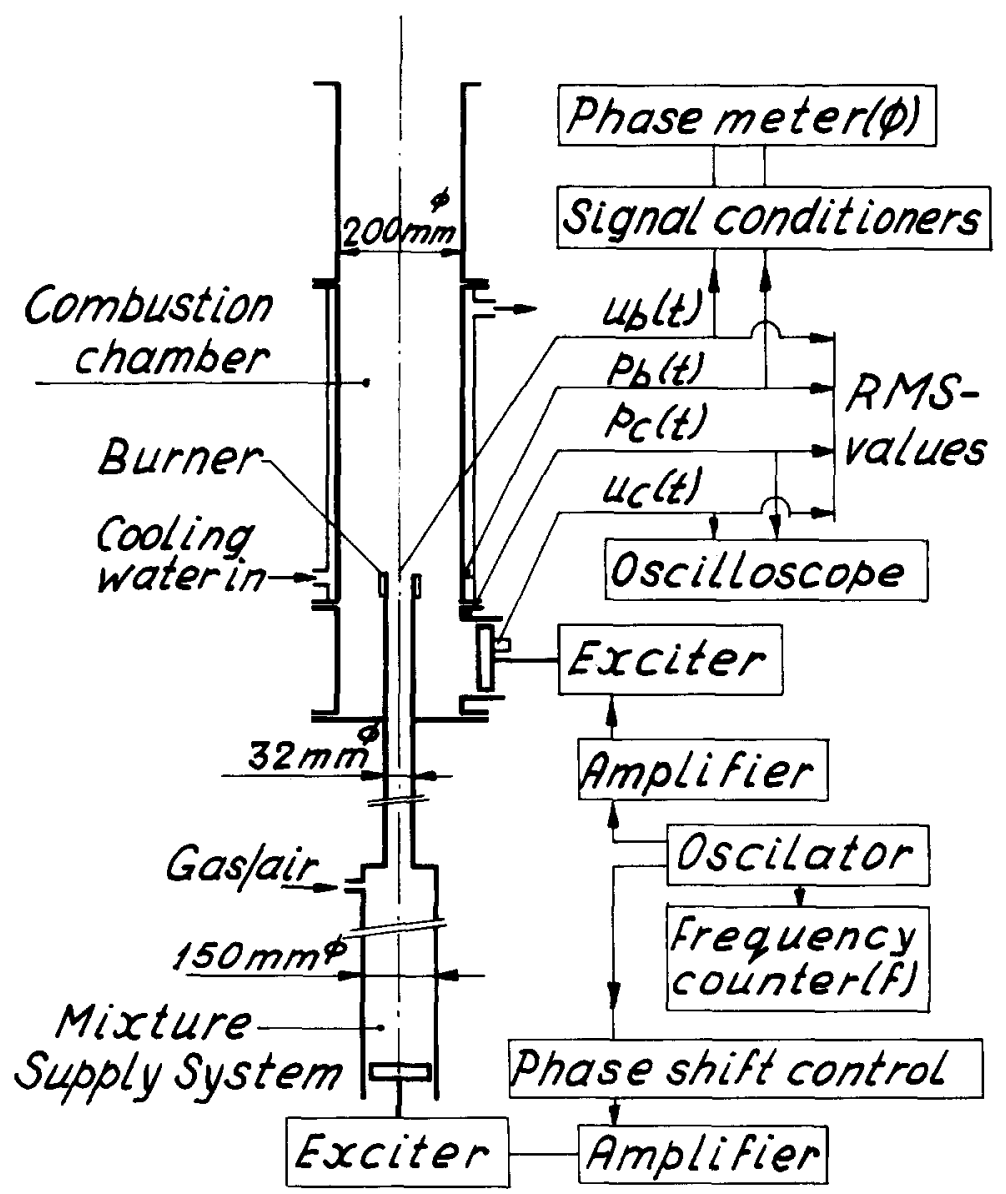

Fig. 1. Experimental apparatus.

ated near the piston to measure the sound pressure, $p_{\mathrm{c}}(t)$, in front of the piston. The second transducer is for measuring the sound pressure, $p_{\mathrm{b}}(t)$, in the plane of the burner face. The piston velocity, $u_{\mathrm{c}}(t)$, is measured by an accelerometer mounted on the piston.

A constant temperature hot wire anemometer is used to monitor velocity fluctuations, $u_{\mathrm{b}}(t)$, of the gas mixture at the burner face. As this probe is placed upstream in the mixture supply (Fig. 2), it has to be calibrated by a second probe, placed at the burner face, without a flame.

The premixed, turbulent, propane-air flame is stabilized by pilot flames at the burner face. The fuel-air ratio is stoichiometric. The firing rates are given in the table of Fig. 9. The diameter of the combustion chamber is $200 \mathrm{~mm}$. The length of the combustion chamber (quarter wavelength) is different and depends on the frequency at which each experiment is performed. The gas and air flow rates are determined by calibrated nozzles. The mixture supply system is designed in such a way that fluctuations of the mixture flow velocity at the burner face can be excited at a desired amplitude by a second piston at the end of the mixture supply tubes (Fig. 3 ). This velocity fluctuation, $u_{\mathrm{b}}(t)$, and the combustion chamber sound pressure, $p_{\mathrm{b}}(t)$, have the same frequency and can be made to differ in any desired phase angle, $\phi$.

These variables are shown in the vector diagram of Fig. 5. The phase shift, $\phi$, is measured by a phase meter. The mixture supply system is so dimensioned that flow fluctuations at the burner face, caused by the pressure fluctuations, $p_{\mathrm{b}}(t)$, 


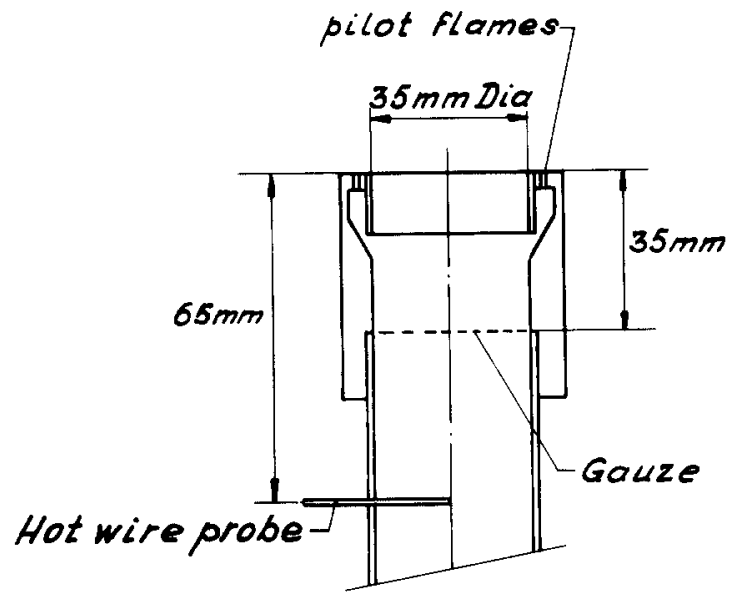

Fig. 2. Single port burner. in the combustion chamber, are prevented. Figure 3 shows this system, consisting of two tubes with diameter jump. This diameter jump acts acoustically as an open end for the tube with the smaller diameter and as a closed end for the tube with the larger diameter. The length of each tube can be varied in order to get a standing wave at the desired frequency. The two pistons, driving the standing waves, are each coupled to an electrodynamic vibration exciter with associated power amplifier.

\section{THE ACOUSTIC POWER BALANCE}

The piston at the bottom of the combustion chamber is driving a standing wave within this combus-

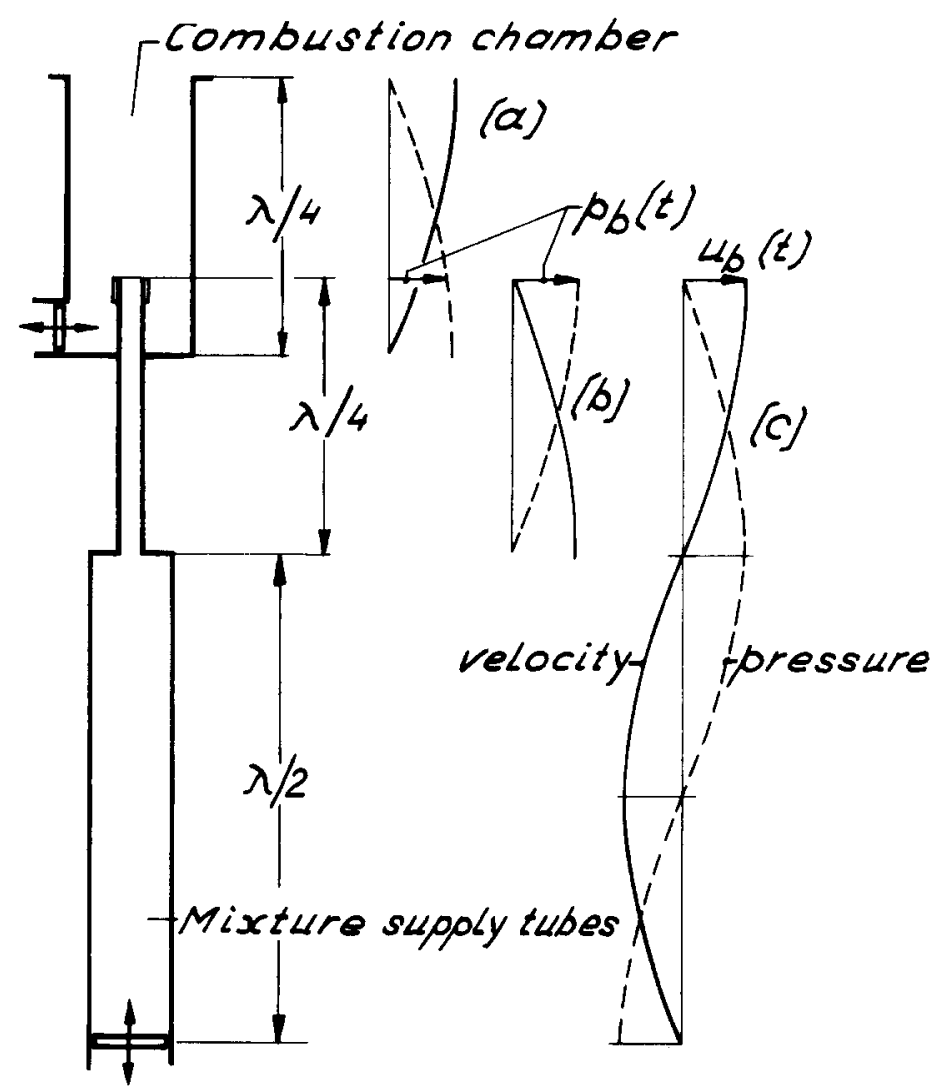

Fig. 3. Geometry of the mixture supply system. (a) Standing wave within the combustion chamber, giving the fluctuating pressure $p_{\mathrm{b}}(t)$ at the burner port. (b) Standing wave within the burner tube, induced by $p_{\mathrm{b}}(t)$. (c) Standing wave, driven by the piston within the mixture supply tubes, with the desired velocity fluctuation $u_{\mathrm{b}}(t)$ at the burner port. 
tion chamber at resonance frequency and at a certain constant sound pressure amplitude. The acoustic power input by this piston is $W_{\mathbf{c}}$. Figure 4 shows the acoustic power input and dissipation in the combustion chamber.

The fluctuating mixture flow velocity, $u_{\mathrm{b}}(t)$, at the burner face will lead to a fluctuating heat release of the flame, resulting in a second input of acoustic power, $W_{f}$, to the standing wave, according to Rayleigh's hypothesis. In this paper our attention is focused on measuring the magnitude of $W_{\mathrm{f}}$. A third sound power input to the standing wave is at the burner face, $W_{\mathrm{b}}$, due to the fluctuating flow velocity, $u_{\mathrm{b}}(t)$.

In the steady rate the power supplied by these sources must equal the acoustic power dissipated in the combustion chamber, $W_{\mathrm{d}}$. Thus we have a power balance

$w_{\mathrm{d}}=w_{\mathrm{c}}+w_{f}+w_{\mathrm{b}}$

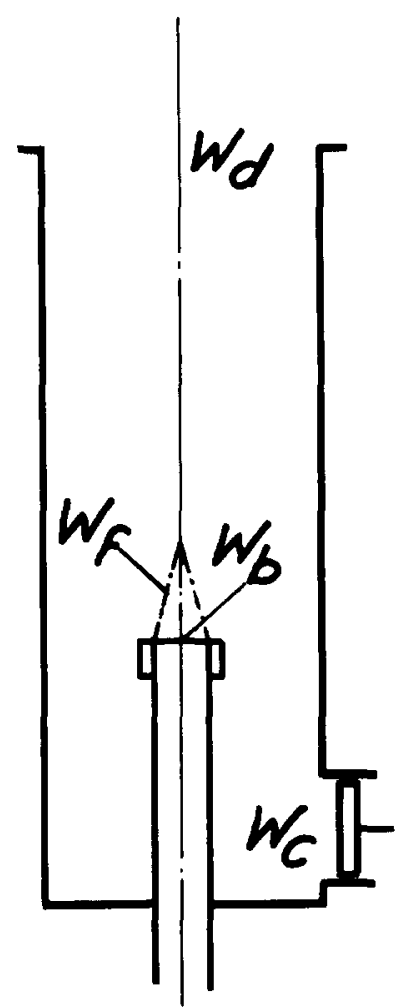

Fig. 4. Acoustic power input and dissipation within the combustion chamber. and so

$W_{\mathrm{f}}=W_{\mathrm{d}}-w_{\mathrm{c}}-W_{\mathrm{b}}$

We can generally state that the rate at which acoustic energy is flowing through a given area is the product of the sound pressure times the inphase component of the volume flow velocity normal to that area. The time average rate of that product is

$W=\frac{A}{T} \int_{0}^{T} p(t) \cdot u(t) d t$

where

$p(t)=\hat{p} \cos \omega t$

$u(t)=\hat{u} \cos (\omega t+\phi)$,

which gives

$W=\frac{1}{2} A \cdot \hat{p} \cdot \hat{u} \cdot \cos \phi$.

This equation may be used for calculating the values of each term in Eq. (1). The power, $\boldsymbol{w}_{\mathbf{c}}$, supplied by the piston to the standing wave in the combustion chamber at resonance frequency can be calculated from

$W_{\mathrm{c}}=\frac{1}{2} A_{\mathrm{c}} \cdot \hat{p}_{\mathrm{c}} \cdot \hat{u}_{\mathrm{c}}$.

The acoustic power supplied to the standing wave at the burner face, $W_{\mathrm{b}}$, can be calculated from

$W_{b}=\frac{1}{2} A_{b} \cdot \hat{p}_{\mathrm{b}} \cdot \hat{u}_{\mathrm{b}} \cdot \cos \phi$.

From Eq. (1) we find that the acoustic power input by the piston, $W_{\mathrm{c}}$, is equal to the power, dissipated in the combustion chamber, $W_{\mathbf{d}}$, when the power input of the flame, $W_{f}$, and at the burner face, $W_{\mathrm{b}}$, both equal zero. This is realized by reducing the velocity fluctuations in the burner port, $\dot{u}_{\mathrm{b}}$, to zero. The sound pressure, $\dot{p}_{\mathrm{b}}$, at the burner port must maintain the same magnitude. This is controlled by the piston velocity, $\hat{u}_{\mathrm{c}}$. The mean caloric heat release is not changed. 
Under these conditions

$W_{\mathrm{d}}=W_{\mathrm{c}}=\frac{1}{2} \cdot A_{\mathrm{c}} \cdot \hat{p}_{\mathrm{c}} \cdot \hat{u}_{\mathrm{c}}$

Now the only unknown quantity in Eq. (1) is the acoustic power input of the flame, $W_{f}$. So we can compute $W_{\mathrm{f}}$ from the measured values of the parameters, $\hat{p}_{\mathrm{c}}, \hat{u}_{\mathrm{c}}, A_{\mathrm{c}}, \phi, \hat{p}_{\mathrm{b}}, \hat{u}_{\mathrm{b}}, A_{\mathrm{b}}$.

The computed values of $W_{\mathrm{f}}$ obtained from such an experiment depend of course on some other parameters, which are: the frequency, the mean heat release, the air-fuel ratio, and the sound pressure amplitude, $\hat{p}_{\mathbf{b}}$.

\section{EXPERIMENTS AND RESULTS}

The experimental program was divided into two parts. In the first part attention was focused on testing the validity of this measuring principle. As the second part of the program, the influence of some relevant parameters was investigated and discussed. The first experiments, not described here, had been performed with a laminar flat flame, because with a turbulent cone-shaped flame no acoustic power release from the flame was found. Only by improving the accuracy of the experiments was it possible to measure the acoustic power of turbulent cone-shaped flames.

\section{Experiments}

Figure 5 shows the results of one experimental run. The acoustic power, $W_{f}$, released from the flame as a function of the phase angle, $\phi$, is almost harmonic. This phase angle, $\phi$, is introduced in the second section. This result is in accordance with Rayleigh's hypothesis, stating that only that part of the fluctuating heat release that is in phase with the sound pressure contributes to the addition of acoustic power. According to Rayleigh's statement, maximum acoustic power supply occurs when the fluctuating heat release and the sound pressure are in phase and also when $\phi=\alpha$ in Fig. 5. Maximum damping occurs when the phase shift is 180 degrees. For one experimental run the frequency can be chosen. From this frequency the wavelength $\lambda$ is calculated. The combustion chamber and the mixture supply tubes are given the lengths according to Fig. 3. After the combustion chamber is warmed up by the flame, it is excited at its resonance frequency. This is the index value of frequency. For this value the flame acoustic power, $W_{f}$, has a maximum (see Fig. 5) where $\phi=\alpha$.

During an experimental run the length of the combustion chamber is not changed, so the resonance frequency will change in accordance with the second statement of Rayleigh's hypothesis; there is an increase or decrease of the oscillation frequency when the fluctuating heat release occurs at the moment when the sound pressure amplitude equals zero. Figure 5 also shows this frequency change.

It is also possible to make an experimental run without changing the frequency. In that case the length of the combustion chamber must be adjusted to the resonance condition for each value of $\phi$.

The experiments show that it is possible to measure quantitatively the acoustic power release of a periodic fluctuating flame. The magnitude and phase shift of the acoustic power will generally depend on the properties and geometry of the fluctuating flame and on the amplitude of the sound pressure around the flame.

\section{Dependence on Sound Pressure Amplitude}

The amplitude of the sound pressure near the burner face can be controlled by the piston at the bottom of the combustion chamber. The acoustic power supply of the flame is measured with six amplitudes of the sound pressure, ranging from $100 \mathrm{~N} / \mathrm{m}^{2}$ to $600 \mathrm{~N} / \mathrm{m}^{2}$. Each experiment gives a result like Fig. 5, the maximum value of which is plotted in Fig. 6. Within experimental error there is a linear increase in the value of the acoustic power with increase in sound pressure amplitude.

This result can be explained by an analogous situation of a volume of air $V$ in a cylinder with a piston. Let the pressure of that air be the ambient pressure, $p_{0}$. When heat is added to that volume of air, at constant pressure, the density is reduced. This causes an expansion of that volume from $V$ to $V+\Delta V$. The product of this expansion 


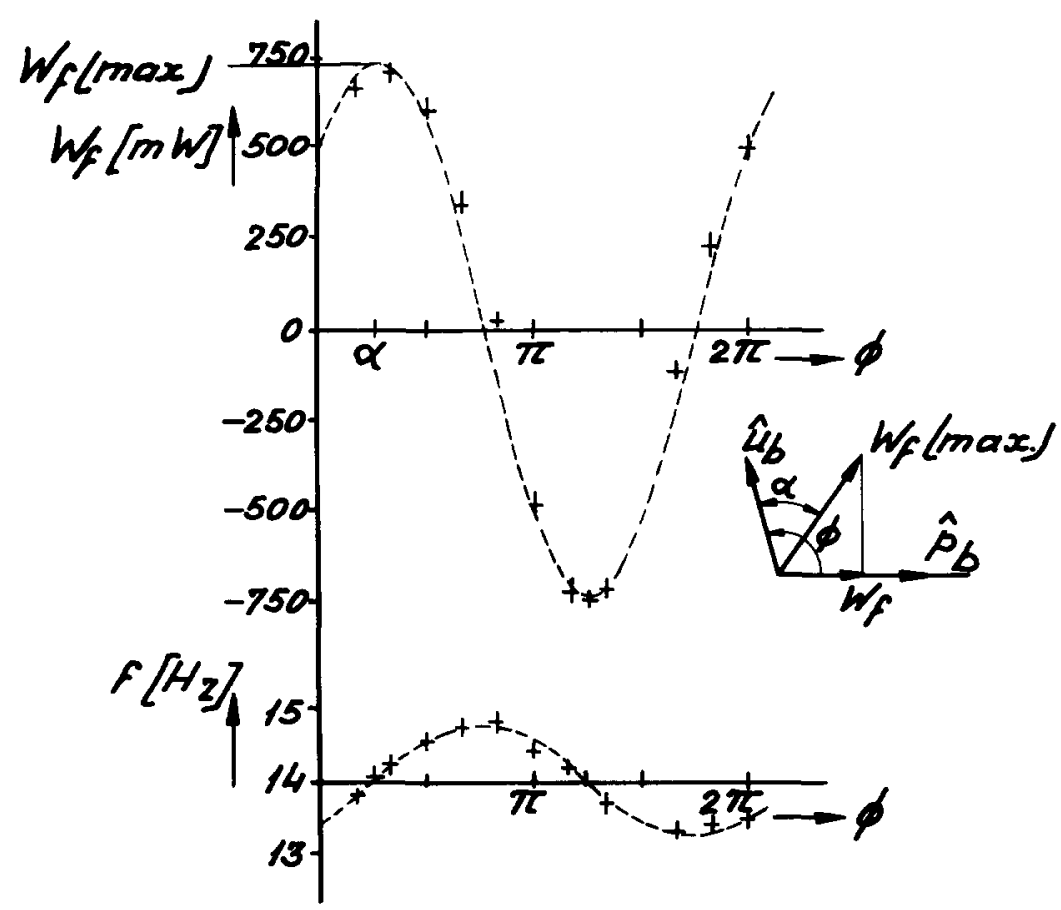

Fig. 5. Results from one experimental run. The acoustic power input from the flame $W_{\mathrm{f}}$ and the frequency $f$ as a function of the phase shift $\phi$. Dotted line is a sine function. $\bar{u}_{\mathrm{b}}=2.30 \mathrm{~m} / \mathrm{sec}, \hat{u}_{\mathrm{b}}=0.5 \mathrm{~m} / \mathrm{sec}, \hat{p}_{\mathrm{b}}=400 \mathrm{~N} / \mathrm{m}^{2}$.

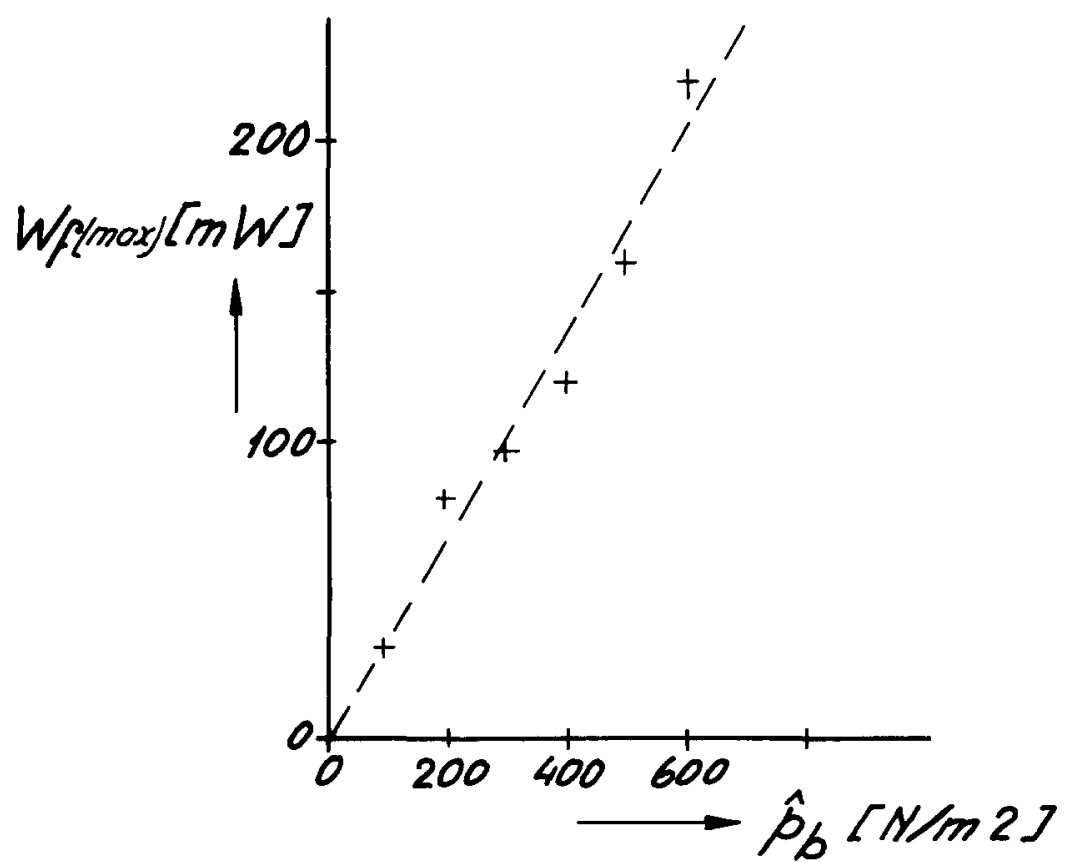

Fig. 6. Variation of the amplitude of the acoustic power with sound pressure amplitude. $\bar{u}_{\mathrm{b}}=2.60 \mathrm{~m} / \mathrm{sec}, \hat{u}_{\mathrm{b}}=0.5 \mathrm{~m} / \mathrm{sec} . f=120 \mathrm{~Hz}$. 
$\Delta V$ times the pressure $p_{0}$ is the amount of work, $E$, done by the expanding air

$$
E=p_{0} \cdot \Delta V .
$$

The thermodynamic expression for this work is

$E=\frac{\gamma-1}{\gamma} \cdot Q$.

Where $Q$ is the amount of heat addition into that volume of air (ideal gas with constant specific heat, reversible process). Using these two equations, we obtain

$\Delta V=\frac{\gamma-1}{\gamma} \cdot \frac{Q}{p_{0}}$

This thermal expansion is also the basic mechanism driving a standing wave. Lord Rayleigh stated that in order to drive oscillations, heat must be added at the moment of greatest compression. In that case the acoustic energy supplied to the standing wave is the product of the sound pressure times the thermal expansion (see the third section).

In the combustion chamber there is a constant mean heat flow from the flame to the wall of the combustion chamber. The fluctuating heat release imposed on the mean heat release is caused by the fluctuating component of the mixture flow velocity, $u_{\mathrm{b}}(t)$, and gives the thermal expansion driving the standing wave. This fluctuating component of the heat release, $q(t)$, is a linear function of the fluctuating velocity, $u_{\mathrm{b}}(t)$.

$q(t)=($ const. $) \cdot u_{b}(t)$

Using Eq. (3) for the thermal expansion, we obtain

$\Delta V(t)=\frac{\gamma-1}{\gamma} \cdot \frac{q(t)}{p_{0}}=$ (const.) $\cdot \frac{\gamma-1}{\gamma} \cdot \frac{u_{\mathrm{b}}(t)}{p_{0}}$.

The sound pressure of the standing wave near the flame is $p_{\mathrm{b}}(t)$. By analogy with this model, the acoustic energy released by the flame is

$E=\frac{\text { (const.) }}{p_{0}} \cdot \frac{\gamma-1}{\gamma} \cdot u_{\mathrm{b}}(t) \cdot p_{\mathrm{b}}(t)$.
On integrating this equation, we obtain an expression for the acoustic power of the flame

$W_{f}=$ (const. $) \cdot u_{\mathrm{b}} \cdot p_{\mathrm{b}}$

The experimental results are in accordance with this equation, showing that there is a linear function relating $W_{\mathrm{f}}$ to the product $\left(\hat{u}_{\mathrm{b}} \cdot \dot{p}_{\mathrm{b}}\right)$.

\section{Dependence on Velocity Amplitude}

The standing wave within the mixture supply system causes the fluctuating velocity $u(t)$ at the burner face. The dependence of the acoustic power on the amplitude of this fluctuating velocity is shown in Fig. 7 for two different mean flow velocities.

Within experimental error, these results demonstrate a linear increase in the value of the acoustic power with increase in the velocity amplitude of the mixture supply. This is also explained by Eq. (4).

\section{Dependence on Flame Shape}

Figure 8 shows sketches of periodically varying flames at three different frequencies, caused by the fluctuating component of the mixture flow velocity at the burner face.

The height, $h$, of the flame depends on the mean mixture flow velocity, $\bar{u}_{\mathrm{b}}$. The table of Fig. 9 shows the measured values. This flame height is measured "by eye." The fluctuating component of the velocity propagates from the burner face to the tip of the flame as a traveling wave, of which the wavelength is $\Lambda=\frac{\bar{u}_{\mathrm{b}}}{f}$.

When the height of the flame is larger than half this wavelength, there is a self-canceling effect, as can be seen in Fig. 8(c). So the acoustic power of the flame will decrease when the frequency increases, keeping the other relevant parameters at constant values. Thus the acoustic power depends 


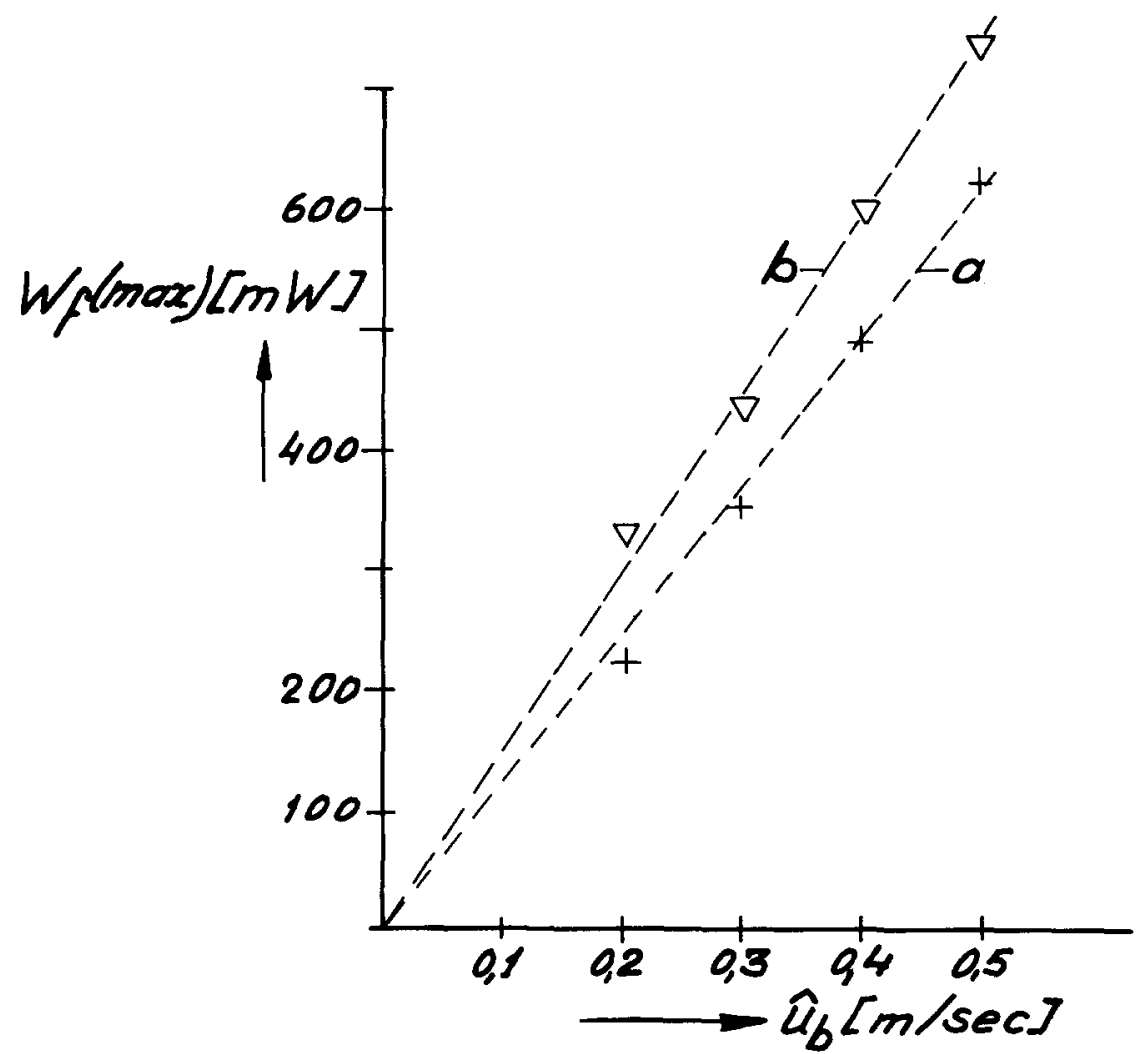

Fig. 7. Variation of the amplitude of the acoustic power with velocity amplitude. $\hat{p}_{\mathrm{b}}=$ $400 \mathrm{~N} / \mathrm{m}^{2}, f=15 \mathrm{~Hz}$. (a) $\bar{u}_{\mathrm{b}}=3.50 \mathrm{~m} / \mathrm{sec},(\mathrm{b}) \bar{u}_{\mathrm{b}}=2.30 \mathrm{~m} / \mathrm{sec}$.

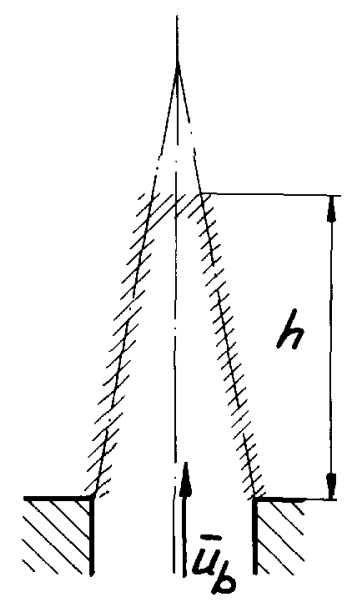

(a)

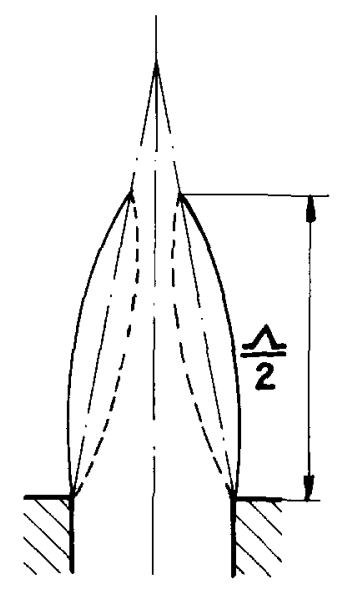

(b)

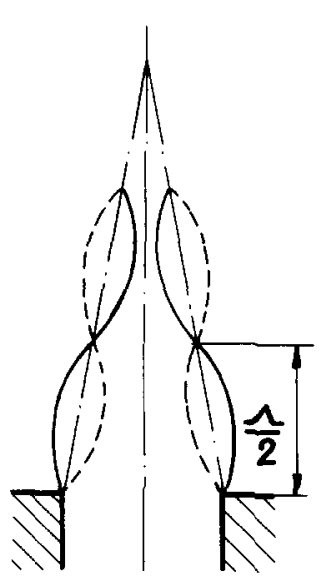

(c)

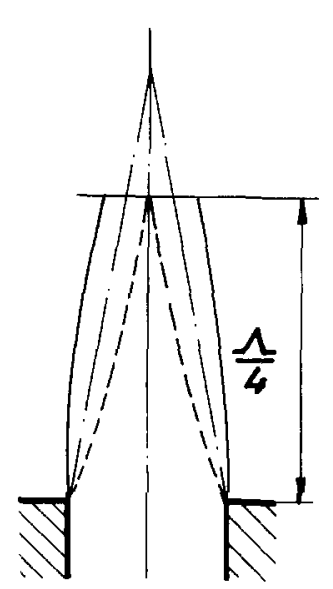

(d)

Fig. 8. Sketches of fluctuating flame. (a) Geometry of turbulent flame; (b), (c) and (d) idealized oscillating flame. The frequency of flame (c) is two times the frequency of flame (b). 


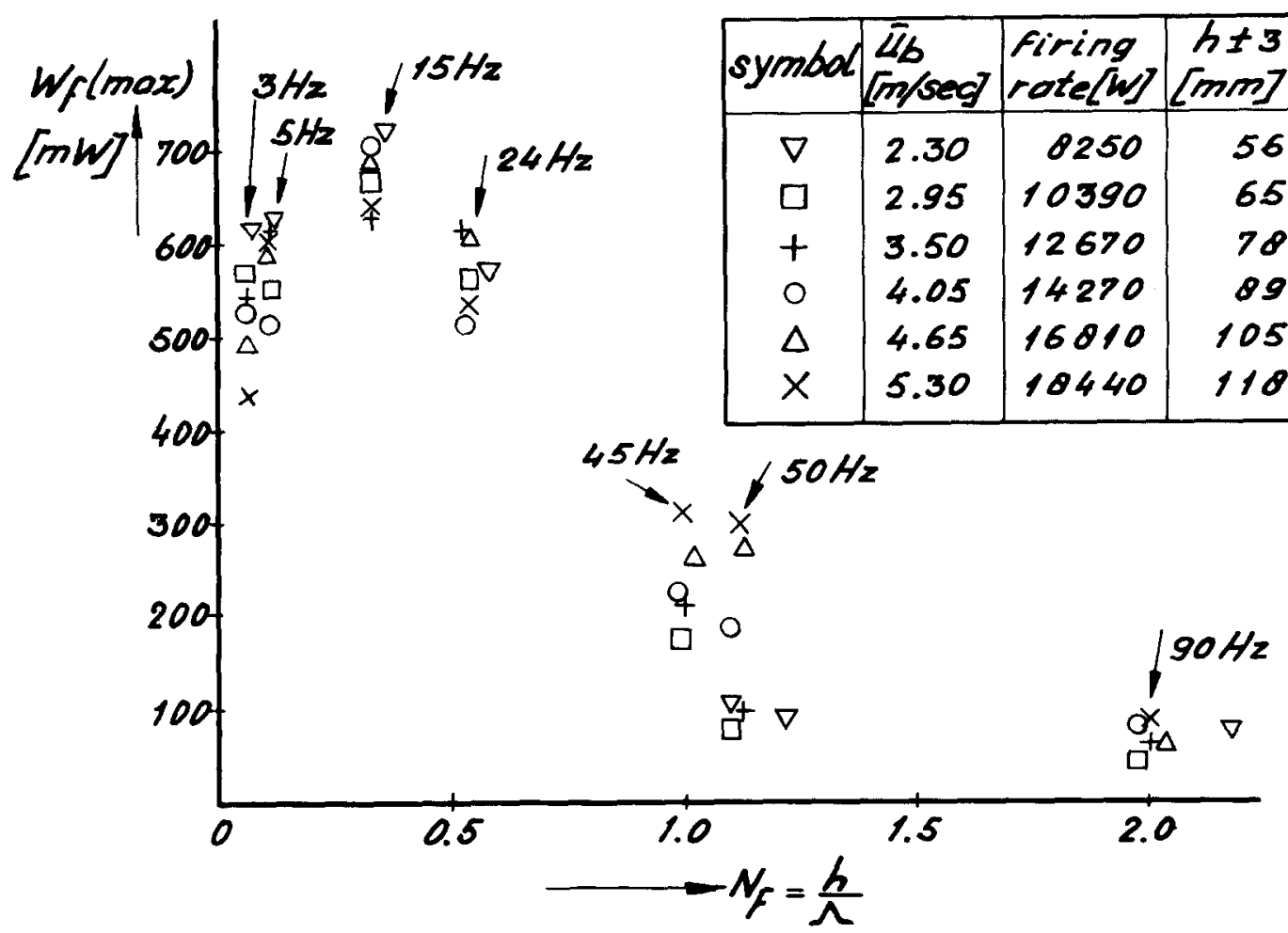

Fig. 9. Flame acoustic power as a function of the flame shape. $\hat{u}_{\mathrm{b}}=0.5 \mathrm{~m} / \mathrm{sec}, \hat{p}_{\mathrm{b}}=$ $400 \mathrm{~N} / \mathrm{m}^{2}$.

on a flame shape number, $N_{\mathrm{f}}$, where

$$
N_{f}=\frac{h}{\Lambda}=\frac{h f}{\bar{u}_{\mathrm{b}}} .
$$

The variation of the acoustic power with the flame shape number obtained by experiment is shown in Fig. 9. There is no reason to suppose that there will be gaps in the data. These experiments show a maximum for $N_{\mathrm{f}} \cong 0.25$. In this situation the idealized shape of the flame is as shown in Fig. 8(d).

The acoustic power, released by the flame $W_{\mathrm{f}}(\max )$, decreases for larger values of $N_{\mathrm{f}}$. This is caused by the self-canceling effect already described. The decrease of the acoustic power for values of $N_{\mathrm{f}}$ smaller than $N_{\mathrm{f}} \cong 0.25$ is not yet explained.

Putnam [1] introduced a driving potential as a function of $f \cdot(h / 3) / \bar{u}_{\mathrm{b}}$ (for long flames relative to the distance between the base of the flame and the burner face). The theoretical derivation of this potential shows a maximum for $f \cdot(h / 3) / \bar{u}_{\mathrm{b}}=0.18$ and for $N_{\mathrm{f}}=0.54$.

\section{CONCLUSIONS}

1. Experiments in a laboratory combustion chamber showed that it is possible to measure the magnitude of the acoustic power of a fluctuating flame supplied to a standing wave of a combustion driven oscillation. It was possible to measure the dependence on some relevant parameters.

2. There is a linear increase in the value of the acoustic power with increase in sound pressure amplitude of the standing wave.

3. The experiments show a linear increase in the value of the acoustic power with increase in the amplitude of the fluctuating fuel-air flow velocity. 
4. The experiments show that the acoustic power of the flame decreases for increasing oscillation frequency. This is explained by means of a self-canceling effect of the flame. The maximum of the acoustic power occurs when $h \cdot f / \bar{u}_{\mathrm{b}}=0.25$, when the frequency is about $10 \mathrm{~Hz}$. Measurements at smaller frequencies down to $f=3 \mathrm{~Hz}$ show a small decrease of the acoustic power.

\section{NOMENCLATURE}

$\begin{array}{ll}A & \text { area } \\ E & \text { energy } \\ f & \text { frequency } \\ h & \text { flame height } \\ N_{\mathrm{f}} & \text { flame shape number } \\ p(t) & \text { sound pressure } \\ \hat{p} & \text { sound pressure amplitude } \\ p_{0} & \text { ambient air pressure } \\ Q & \text { heat } \\ q(t) & \text { fluctuating component of heat } \\ & \text { release } \\ T & \text { oscillation period } \\ t & \text { time } \\ u(t) & \text { fluctuating velocity } \\ \hat{u} & \text { velocity amplitude }\end{array}$

$\mathrm{m}^{2}$

$\mathrm{J}$

$1 / \mathrm{sec}$

$\mathrm{m}$

$\mathrm{N} / \mathrm{m}^{2}$

$\mathrm{N} / \mathrm{m}^{2}$

$\mathrm{N} / \mathrm{m}^{2}$

$\mathrm{J}$

J

$\sec$

$\sec$

$\mathrm{m} / \mathrm{sec}$

$\mathrm{m} / \mathrm{sec}$

$\begin{array}{lll}\bar{u} & \text { mean velocity } & \mathrm{m} / \mathrm{sec} \\ V & \text { volume } & \mathrm{m}^{3} \\ \Delta V & \text { volume change } & \mathrm{m}^{3} \\ W & \text { power } & \mathrm{W} \\ \alpha, \phi & \text { phase angle } & \mathrm{rad} \\ \gamma & \text { specific heat ratio } & \\ \lambda, \Lambda & \text { wave length } & \mathrm{m} \\ \omega & \text { angular frequency } & \mathrm{rad} / \mathrm{sec}\end{array}$

\section{Subscripts}

$\begin{array}{ll}\text { b } & \text { burner } \\ \text { c } & \text { combustion chamber } \\ d & \text { dissipation } \\ \text { f } & \text { flame } \\ \text { (max) } & \text { maximum value of the } \\ & \text { acoustic power }\end{array}$

\section{REFERENCES}

1. Putnam, A. A., Combustion Driven Oscillations in Industry, Elsevier, New York, 1971, pp. 26-38.

2. Lord Rayleigh, The Theory of Sound, Dover, New York, Vo. 2, 1945.

3. Roberts, J. P., Combust. Flame 33:79-83 (1978).

Received 17 July 1979; revised 9 July 1980 